\title{
Knowledge of orthopaedic implant costs and healthcare schemes among orthopaedic residents
}

\author{
Chloe Xiaoyun $\underline{\text { Chan }}^{1}$, MBBS, Gen Lin $\underline{\mathrm{FoO}}^{2}$, MBBS, FRCS, Ernest Beng Kee $\underline{K w e k}^{2}$, MBBS, FRCS
}

\begin{abstract}
There is a paucity of available research on knowledge of orthopaedic implant costs and healthcare schemes among orthopaedic residents. With the rising healthcare costs in Singapore, it is imperative for residents, who are the future surgeons, to understand these issues in order to provide proper counselling and cost-effective management. This study aimed to quantify how accurately they understood these issues and determine if senior residents had better knowledge given their increased experience. An online survey was administered to all orthopaedic residents within a residency programme. There was poor knowledge of implant costs and healthcare schemes among residents. Junior residents fared better at healthcare schemes, while senior residents fared better at estimation of implant costs. Education on these issues should be incorporated into the residency programme to bring about more holistic and cost-conscious clinicians.
\end{abstract}

Keywords: healthcare schemes, implant cost, orthopaedic resident, residency programme

\section{INTRODUCTION}

The emerging grey tsunami poses a significant strain on Singapore's healthcare system. In the next few decades, the national healthcare expenditure will continue to rise..$^{(1)}$ In response, several new schemes and modifications were introduced to enable a more affordable healthcare system. These included the Community Health Assist Scheme (CHAS) in 2012, the Pioneer Generation Package in 2014, and amendments to the national medical savings scheme, Medisave, and medical endowment fund, Medifund. In orthopaedic surgery, patients are now allowed to tap on subsidies for computed tomography $(\mathrm{CT})$, magnetic resonance (MR) imaging and outpatient hyperbaric oxygen therapy. With newer technology and implant designs, orthopaedic implant costs have been consistently rising over the years. It is imperative that surgeons have a good understanding of implant costs in order to provide proper counselling, cost-effective management and greater patient satisfaction in care.

Education on these topics has not traditionally been part of the residency training programme in Singapore. Our study therefore aimed to: (a) explore orthopaedic residents' perception of their knowledge of healthcare schemes and implant costs; (b) quantify how accurately residents understood healthcare schemes and implant costs; and (c) determine if senior residents had better knowledge than junior residents given their increased experience.

\section{METHODS}

A cross-sectional 27-item online survey was administered to all orthopaedic surgery residents within two sites of an orthopaedic surgery residency programme. The survey consisted of three components. Respondents first rated their perception of their knowledge about healthcare schemes and orthopaedic implant costs prior to attempting the survey. Options provided included 'none', 'poor', 'average', 'good' and 'excellent'. Next, they answered 14 questions on healthcare schemes in Singapore, based on common questions posed by patients in clinical practice. These included questions on the amount of government subsidy that was allowed for inpatient bills in a public hospital ward, the maximum Medisave withdrawal limit for inpatient charges, and whether Medisave can be used for outpatient CT or MR imaging, vacuum-assisted closure and hyperbaric oxygen therapy. Residents were also quizzed on the qualification criteria for CHAS and the Pioneer Generation Package, and the uses of these new schemes with reference to specialist outpatient clinic bills. The third component assessed respondents' estimation of the costs of 13 common implants in practice. The implants were described in detail, including the size and number of holes of the implant, and the exact quantity of cement.

Respondents were categorised into senior residents (R4 and R5) and junior residents (R1, R2 and R3) for intergroup comparisons. Healthcare scheme scores were represented in percentages (score in percentage $=$ [number of questions answered correctly/14] $\times 100)$. The overestimation rate ([estimated cost - implant cost]/implant cost) was calculated to determine the accuracy of implant cost estimations.

Statistical analysis was performed using IBM SPSS Statistics version 23.0 (IBM Corp, Armonk, NY, USA). Two-sample $t$-test and Mann-Whitney $U$ test were used to compare healthcare scores and overestimation rates of implant costs, respectively, between the senior and junior residents. A p-value $<0.05$ was considered to be statistically significant.

\section{RESULTS AND DISCUSSION}

A total of 26 (78.8\%) out of 33 residents participated in the survey. The 17 junior residents were $R 1(n=2,7.7 \%), R 2(n=6,23.1 \%)$ or R3 $(n=9,34.6 \%)$. The nine senior residents were R4 $(n=4$, $15.4 \%)$ or R5 ( $n=5,19.2 \%)$. The majority of the respondents ( $\mathrm{n}=21,80.8 \%$ ) rated their knowledge on healthcare schemes as 'poor' to 'average'. For the 14 healthcare scheme questions, 
Table I. Healthcare scheme scores and implant cost estimations of residents who took the survey.

\begin{tabular}{|c|c|c|c|c|}
\hline \multirow[t]{2}{*}{ Response } & \multicolumn{3}{|c|}{ No. $(\%) /$ mean \pm standard deviation } & \multirow[t]{2}{*}{ p-value } \\
\hline & Junior $(n=17)$ & Senior $(n=9)$ & Total $(n=26)$ & \\
\hline \multicolumn{5}{|l|}{ Healthcare schemes } \\
\hline Perception of knowledge & & & & - \\
\hline Verypoor & $2(11.8)$ & $1(11.1)$ & $3(11.5)$ & \\
\hline Poor & $7(41.2)$ & $4(44.4)$ & $11(42.3)$ & \\
\hline Average & $8(47.1)$ & $2(22.2)$ & $10(38.5)$ & \\
\hline Good & $0(0)$ & $2(22.2)$ & $2(7.7)$ & \\
\hline Score (\%) & $36.6 \pm 12.6$ & $23.0 \pm 12.3$ & $31.9 \pm 14.9$ & 0.015 \\
\hline \multicolumn{5}{|l|}{ Implant costs } \\
\hline Perception of knowledge & & & & - \\
\hline Verypoor & $3(17.6)$ & $2(22.2)$ & $5(19.2)$ & \\
\hline Poor & $8(47.1)$ & $3(33.3)$ & $11(42.3)$ & \\
\hline Average & $6(35.3)$ & $3(33.3)$ & $9(34.6)$ & \\
\hline Good & $0(0)$ & $1(11.1)$ & $1(3.8)$ & \\
\hline Overestimation rate (\%) & $522.9 \pm 562.4$ & $183.7 \pm 96.8$ & $405.5 \pm 482.2$ & 0.150 \\
\hline
\end{tabular}

junior residents scored an average of $36.6 \% \pm 12.6 \%$ (range $21.4 \%-64.3 \%$ ) and senior residents scored $23.0 \% \pm 12.3 \%$ (range $7.1 \%-42.9 \%$ ), with a combined score of $31.9 \% \pm 14.9 \%$ (range $7.1 \%-64.3 \%$ ). Junior residents were statistically more knowledgeable than senior residents $(p=0.015)$ Details of the survey responses are found in Table I.

Our study demonstrated poor knowledge of healthcare policies among residents. A plausible reason could be the lack of emphasis on acquiring such knowledge in the years of medical school and residency training, and an over-reliance on nurses and administrative staff to handle these topics. Arguably, junior residents might have received a higher mean score because they had recently completed their internship and would have dealt with more administrative and financial issues in patient care, and hence were more familiar with healthcare subsidies compared to senior residents. Based on our results, our traditional learning model needs to be modified to better suit the current healthcare climate, in which cost is an important consideration in the management of patients. Hard copy or online reference materials on healthcare schemes and subsidies can also be made available in clinics for both residents and consultants.

The majority of respondents $(n=20,76.9 \%)$ rated their knowledge on implant costs as poor to average (Table I). The average overestimation rate in junior residents was $522.9 \%$ compared to $183.7 \%$ in senior residents. There was no significant difference between the senior and junior resident groups $(p=0.15)$. Our finding was similar to that of Streit et al, who established that residents had a higher mean percentage error in implant cost estimations compared to attending surgeons (73\% vs. 59\%). ${ }^{(2)}$ This suggests that level of seniority does affect estimations of implant costs.

Junior residents were most accurate at estimating the cost of the $\mathrm{M} / \mathrm{L}$ taper hip hemiarthroplasty stem and distal radius variable-angle locking compression plate, while the senior resident group most accurately estimated the cost of the $M / L$ taper hip hemiarthroplasty stem and proximal femoral nail antirotation (Table II). The implication is that familiarity with these implants, which are routinely used by residents, improves cost estimations. This finding concurs with Okike et al's study. ${ }^{(3)}$

Both groups were least accurate in their estimation of the two lowest-priced implants, the six-hole $1 / 3$ tubular plate and six-hole limited-contact dynamic compression plate, with a combined average overestimation rate of $2,834.5 \%$ and $1,139.0 \%$ respectively (Table II). Okike et al reported the same observation, that surgeons tended to overestimate the price of low-cost devices. ${ }^{(3)}$

The great disparity between the residents' impressions and the actual cost is alarming. The educational gaps in teaching cost-consciousness should be bridged and inculcated as part of the residency curriculum. It has been proven that providing cost data for tests influences ordering behaviour and knowledge in physicians. ${ }^{(4)}$ Hence, residency programmes should recognise this knowledge gap and improve awareness of implant costs. This can be actively raised as a discussion point during clinical teaching encounters, perioperative case discussions and case log reviews.

Rising healthcare costs is a complex problem that needs to be addressed at all levels of the healthcare industry. While an emphasis on providing cost-conscious care in the education of future surgeons could represent a step in the right direction, we acknowledge that it is but one of many approaches required to tackle this issue.

This study had several limitations. Firstly, it was conducted in two tertiary hospitals, with a training programme that was arguably different compared to other residency programmes. Secondly, we were limited by the relatively small number of residents in the programme. However, we had a relatively high response rate of $78.8 \%$ and higher absolute numbers compared to other studies. ${ }^{(2,5)}$ Lastly, we did not include senior surgeons in the study although knowledge of healthcare schemes and implant costs are relevant to them as well. Instead, we chose to focus on 
Table II. Overestimation rate for each device measured for all respondents.

\begin{tabular}{|c|c|c|c|}
\hline \multirow[t]{2}{*}{ Device } & \multicolumn{3}{|c|}{ Mean overestimation rate (\%) } \\
\hline & Junior $(n=17)$ & Senior $(n=9)$ & Total $(n=26)$ \\
\hline 6-hole $1 / 3$ tubular plate & $3,791.1$ & $1,027.6$ & $2,834.5$ \\
\hline 6-hole 3.5-mm LC-DCP plate & $1,423.0$ & 603.0 & $1,139.0$ \\
\hline 6-hole 3.5-mm LCP plate & 659.8 & 344.4 & 508.2 \\
\hline Anterior cervical plate (CSLP) & 294.5 & 248.6 & 278.0 \\
\hline Exeter hip hemiarthroplasty stem & 176.8 & 49.7 & 132.8 \\
\hline Dynamic hip screw & 151.7 & 27.8 & 108.8 \\
\hline Cement (with gentamicin) & 112.9 & 49.2 & 95.5 \\
\hline USS-II polyaxial pedicle screw & 44.1 & 62.7 & 45.9 \\
\hline Versys Advocate hip hemiarthroplasty stem & 40.8 & 29.0 & 37.2 \\
\hline Distal radius VA-LCP plate & 21.7 & 52.8 & 32.5 \\
\hline Primary total knee arthroplasty & 30.0 & 20.0 & 26.0 \\
\hline M/L taper hip hemiarthroplasty stem & 20.8 & -1.5 & 13.1 \\
\hline PFNA & 25.9 & -15.3 & 11.6 \\
\hline
\end{tabular}

CSLP: cervical spine locking plate; LC-DCP: limited-contact dynamic compression plate; LCP: locking compression plate; PFNA: proximal femoral nail antirotation; VA-LCP: variable-angle locking compression plate

junior doctors, as we wanted to identify gaps in the education of residents.

In conclusion, our residents demonstrated poor knowledge of implant costs and healthcare schemes. Senior residents fared better at the estimation of implant costs and poorer at healthcare scheme questions compared to their junior counterparts. Overestimation rates were as high as 10-30 times of the actual implant cost for the rudimentary implants. These findings highlighted the need for education on these issues, especially in terms of healthcare assistance schemes, within the residency programme to bring about more holistic and cost-conscious clinicians.

\section{REFERENCES}

1. Hansen E, Bozic KJ. The impact of disruptive innovations in orthopaedics. Clin Orthop Relat Res 2009; 467:2512-20.

2. Streit JJ, Youssef A, Coale RM, Carpenter JE, Marcus RE. Orthopaedic surgeons frequently underestimate the cost of orthopaedic implants. Clin Orthop Relat Res 2013; 471:1744-9.

3. Okike K, O'Toole RV, Pollak AN, et al. Survey finds few orthopedic surgeons know the costs of the devices they implant. Health Aff (Millwood) 2014; 33:103-9.

4. Post J, Reed D, Halvorsen AJ, Huddleston J, McDonald F. Teaching high-value, cost-conscious care: improving residents' knowledge and attitude. Am J Med 2013; 126:838-42.

5. Rohman L, Hadi S, Whitwell G. Surgeons' knowledge about the costs of orthopaedic implants. J Orthop Surg (Hong Kong) 2014; 22:221-3. 